\title{
Simulation of Pneumoperitoneum for Laparoscopic Surgery Planning
}

\author{
J. Bano ${ }^{1,2}$, A. Hostettler ${ }^{1}$, S.A. Nicolau ${ }^{1}$, S. Cotin ${ }^{3}$, C. Doignon ${ }^{2}$, H.S. Wu ${ }^{4}$, \\ M.H. Huang ${ }^{4}$, L. Soler ${ }^{1}$, and J. Marescaux ${ }^{1}$ \\ 1 IRCAD, Virtual-Surg, Place de l'Hopital 1, 67091 Strasbourg Cedex, France \\ 2 LSIIT (UMR 7005 CNRS), University of Strasbourg, Parc d'Innovation, \\ Boulevard S. Brant, BP 1041267412 Illkirch Cedex, France \\ 3 SHACRA Group, Inria, France \\ 4 IRCAD Taiwan, Medical Imaging Team, 1-6 Lugong Road, Lukang 505, Taiwan \\ jordan.bano@etu.unistra.fr
}

\begin{abstract}
Laparoscopic surgery planning is usually realized on a preoperative image that does not correspond to the operating room conditions. Indeed, the patient undergoes gas insufflation (pneumoperitoneum) to allow instrument manipulation inside the abdomen. This insufflation moves the skin and the viscera so that their positions do no longer correspond to the preoperative image, reducing the benefit of surgical planning, more particularly for the trocar positioning step. A simulation of the pneumoperitoneum influence would thus improve the realism and the quality of the surgical planning. We present in this paper a method to simulate the movement of skin and viscera due to the pneumoperitoneum. Our method requires a segmented preoperative 3D medical image associated to realistic biomechanical parameters only. The simulation is performed using the SOFA simulation engine. The results were evaluated using computed tomography $[\mathrm{CT}]$ images of two pigs, before and after pneumoperitoneum. Results show that our method provides a very realistic estimation of skin, viscera and artery positions with an average error within $1 \mathrm{~cm}$.
\end{abstract}

Keywords: simulation, surgical planning, pneumoperitoneum.

\section{Introduction}

\subsection{Clinical Context}

Laparoscopic surgery has become a common procedure in the sugical community. It involves inserting an endoscopic camera and several instruments through small incisions made on the abdomen. In order to create a working space, gas is injected into abdominal cavity (pneumoperitoneum). Surgical planning is usually performed on a preoperative image, or in the best case, using a three-dimensional model. Part of the planning requires determining the trocar positioning so that the surgeon has a good triangulation between his instruments as well as good camera viewpoint. However, the preoperative model does not take deformation 
due to gas injection into consideration. Since the pneumoperitoneum shifts the skin up and moves the abdominal viscera by several centimeters [9], the planned trocar positioning becomes inconsistent with the true position in the operating room, which drastically reduces the usefulness of the preoperative planning.

In this paper, we propose a individualized simulation of pneumoperitoneum and viscera motion using a preoperative medical image (CT) that can be used for laparoscopic surgery planning. The realism of the simulation method is evaluated with ground truth data and we show our method can even predict real pneumoperitoneum with an accuracy close to $0.5 \mathrm{~cm}$ which is accurate enough for trocar positioning planning. We highlight that in case the $\mathrm{CT}$ is injected with contrast medium, the abdominal wall arteries (cf. Fig. 1 for abdominal wall artery definition) are visible in the CT image and their position after pneumoperitoneum can also be simulated. This information can be crucial to avoid injury of abdominal wall arteries during trocar insertion, which is a common complication in laparoscopic surgery [2 1074].

\subsection{Previous Work}

To our knowledge, only Kitasaka et al. 35. have explored pneumoperitoneum simulation for surgical planning. They simulated the deformation due to gas injection by applying forces that seem to be antero-posterior, on the inner surface of a portion of the abdominal wall. Recently, they evaluated the accuracy of their results on eight patients by comparing thirteen skin landmark positions after pneumoperitoneum and with simulation results [6]. Their simulation method uses two parameters which are empirically chosen to fit the in vivo data. With an optimally chosen set of parameters, the minimal mean error obtained was $13.8 \mathrm{~mm}$. Although their simulation provides interesting results, its usefulness is still limited since they do not take the viscera motion into account. Moreover the parameters of their model have no biomechanical meaning and cannot be adapted for a particular patient shape.

Soler et al. [1] have presented a patient-specific simulator for laparoscopic surgery. However in this paper, skin and viscera motions due to gas injection are also not taken into account. Viscera shape corresponds to viscera segmentation in the preoperative image (without pneumoperitoneum), and trocar positions are chosen without being related to skin position after pneumoperitoneum. Therefore, integrating realistic skin simulation after pneumoperitoneum would improve the realism of such simulator.

This paper proposes a method to simulate a pneumoperitoneum from a preoperative image, using realistic biomechanical parameters. In Section 2, we present the anatomical structures that we need to model from the preoperative image and how we choose the biomechanical parameters. In Section 3. we explain how the influence of gas pressure on the abdominal cavity is modeled and how we account for multiple contacts between moving structures in the abdomen. Finally, we evaluate in Section 4 our simulation realism using porcine data and we show our simulation accuracy is within $4 \mathrm{~mm}$ on average for the abdominal wall and viscera and $5 \mathrm{~mm}$ for abdominal wall arteries. 


\section{Model Generation}

The input model of our simulation is created from an abdo-thoracic CT in three steps: segmentation, mesh generation and mechanical parameterization.

Segmentation Step. The preoperative image is segmented by dividing it into three regions: the abdo-thoracic wall, the abdominal viscera and the thoracic viscera (Fig. 1). In case they are visible, the arteries in the abdominal wall are also segmented. Note that they are not mandatory for the model. These segmentations are done semi-manually using custom-developed software and take one hour on average. Improvements are planned in future works to reduce the required time.

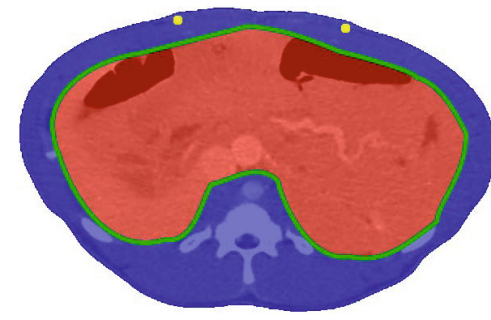

(a) Axial slice

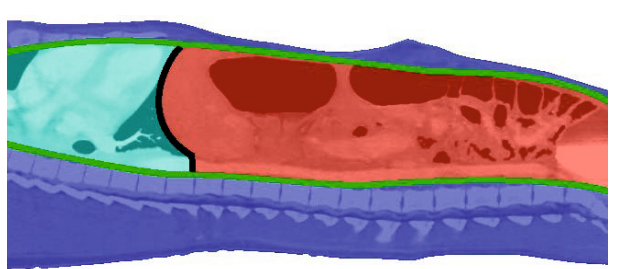

(b) Sagittal slice

Fig. 1. On these two slices extracted from a preoperative pig CT acquisition, one can see the anatomical structures of our simulation model: the abdo-thoracic wall (blue), its internal surface (green line), the abdominal viscera (red), the thoracic viscera (cyan) and the diaphragm (black line). On the left figure, the abdominal wall arteries, located in the abdo-thoracic wall, are colored in yellow.

Mesh Generation. Volume and surface meshes are generated using the CGAL library 11 for the abdo-thoracic wall, the abdominal viscera and the thoracic viscera. Volumetric meshes are required for the finite element approach used in the computation of soft tissue deformation, while surface meshes are used for collision detection. The mesh generation needs a few seconds only from the segmented images.

Mechanical Parameters. Our simulation is based on a finite-element approach using a geometrically non-linear elastic law and a co-rotational formulation. The simulation is parametrized by associating Young's modulus [YM] and Poisson ratio to each volume mesh: the abdominal wall volume mesh is associated with a YM equal to $24 \mathrm{kPa}$ (cf. Song et al. paper [12) and a Poisson ratio of 0.49 (we assume that the abdominal wall is almost incompressible). Although abdominal viscera contain various organs with different stiffnesses, we chose, for simplicity reasons, to consider it as a homogeneous set associated to standard

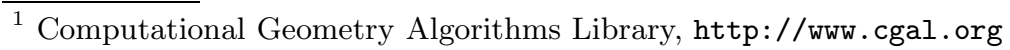


YM of liver (15 kPa found in [8]). The thoracic viscera, containing the lungs and the heart (which reduces the mobility of the diaphragm), was assumed as a homogeneously elastic set ( $\mathrm{YM}$ of $7 \mathrm{kPa}$ ). Moreover, a diaphragm was added to the model with a tenfold YM as for the abdominal wall.

\section{Simulation}

Our approach consists in simulating the realistic phenomena occuring during pneumoperitoneum. Our simulation is performed with SOFA [1] which provides a set of methods for modeling and simulating soft tissues and their interactions. We describe here only the main elements of the simulation, in particular gravity (and its compensation) and the effect of gas pressure in the abdominal cavity.

Mapping and Collision. For our simulation, we use an intricate combination of surface and volumetric meshes. Surface meshes are used for visualization, collision detection or for applying pressure forces. To propagate the information from a surface mesh to a volumetric mesh (or the other way around) we rely on the mapping mechanism provided by SOFA. The basic idea is that each vertex of a surface mesh is associated with a tetrahedron of the volume mesh. If a displacement is applied on a node of the "master" mesh, then a corresponding displacement is propagated to the "slave" mesh (practically, the displacement of a node is distributed to the vertices of the element it maps, according to its barycentric coordinates within that element). Similarly, if a force is applied on a node of a mapped mesh, then the force is propagated to the "master" mesh and distributed over the nearby nodes. The relationship between the initial and mapped forces is given by the transpose of the Jacobian of the mapping: $f_{\text {initial }}=J^{T} f_{\text {mapped }}$

Inverse Gravitational Deformation. To accurately simulate the deformation of the abdominal wall, or to correctly handle contacts between various anatomical structures, we need to include gravitational forces in our simulation. However, the different meshes used in the simulation are extracted from a CT image that has been acquired while gravity was applied to the tissues. Therefore, we cannot naively apply gravitational forces again onto the anatomical models. Although this problem is common in patient-specific simulations, it is not often mentioned and compensated except for some works, as that of Whiteley [13].

The main idea is to create a model representing the patient if he was not subject to gravity. Then, gravity is applied onto this model in order to obtain a new model, including the constraints due to gravity, and having a shape as similar as possible to the preoperative model.

The model without gravity is obtained by applying a negative gravity on the preoperative model. Therefore, a negative gravity is applied while the model is fixed on the table via the contact surface between the table and the pig. 
Gas Pressure. A small part of the posterior skin is fixed all along the craniocaudal direction to simulate the contact with the operating table (Fig. 2). Then, the simulation of the abdominal wall lift is done by applying forces on the abdominal wall internal surface. The force value corresponds to the gas injection pressure of $12 \mathrm{mmHg}$ commonly used in laparoscopic surgery and which direction is normal to the surface. The abdominal wall volume mesh is then expanded due to its mapping, resulting in deformations of its associated surface mesh. Moreover, the abdominal wall internal surface collides with the thoracic viscera surface mesh, which is pushed in the cranial direction. The simulation takes about 30 seconds (Intel Core i7 920 2.67GHz).

Abdominal Wall Artery Simulation. The artery surface mesh is mapped with the abdo-thoracic volume mesh. Therefore, it will be consistently deformed, following the deformation of the abdo-thoracic mesh due to the simulated pressure in the abdominal cavity.

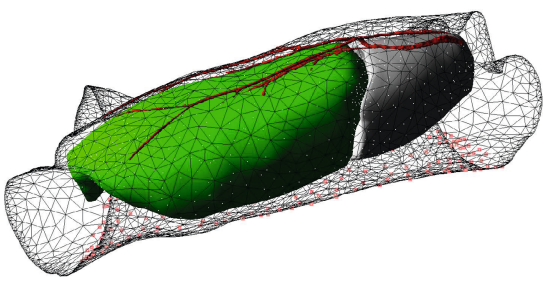

(a) Before simulation

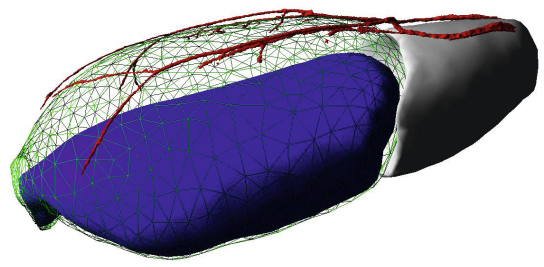

(b) After simulation

Fig. 2. One can see our pneumoperitoneum simulation with skin (black wireframe, on the left only), abdominal cavity (green), thoracic viscera (white), abdominal viscera (blue) and abdominal wall arteries (red). On the left figure, constrained vertices are colored in pink.

\section{Evaluations on Porcine Data}

The assessement of our method was performed using two sets of CT images, acquired before and after creating a pneumoperitoneum for each pig. To evaluate the quality of our simulation, we compare the simulated abdominal wall, viscera and artery positions to the actual ones extracted from the CT data after pneumoperitoneum.

\subsection{Evaluation of the Abdo-Thoracic Wall and Abdominal Viscera Position Simulation}

For each simulated structure, the corresponding ground truth is a very dense point cloud extracted from the binary segmented mask of the CT image after 
Table 1. The euclidian distance existing between each vertex of the simulated surface meshes and the actual ones is computed and sorted in four groups. Each group contains a quartile of the vertex total number.

\begin{tabular}{|c|c|c||c|}
\hline Skin $(\mathrm{mm})$ & Internal surface $(\mathrm{mm})$ & Abdominal viscera $(\mathrm{mm})$ & Color range \\
\hline $0-1.3$ & $0-1.0$ & $0-0.6$ & Blue to Turquoize \\
$1.3-2.7$ & $1.0-2.4$ & $0.6-1.5$ & Turquoize to Green \\
$2.7-5.1$ & $2.4-4.9$ & $1.5-3.8$ & Green to Yellow \\
$5.1-10.6$ & $4.9-16.5$ & $3.8-20.1$ & Yellow to Red \\
\hline \hline $3.2( \pm 2.2)$ & $3.4( \pm 3.0)$ & $2.9( \pm 3.5)$ & Mean ( \pm Std.Dev. $)$ \\
\hline
\end{tabular}

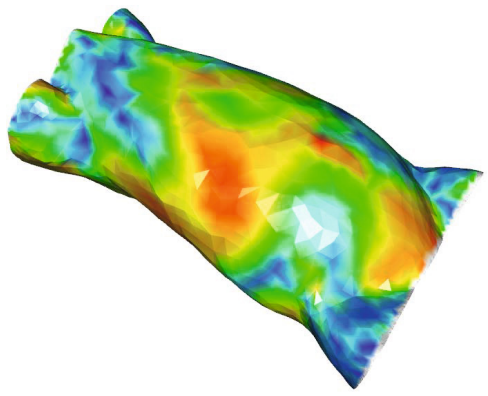

(a) Skin

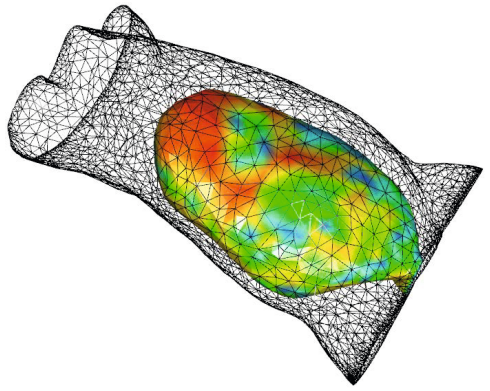

(c) Abdominal viscera

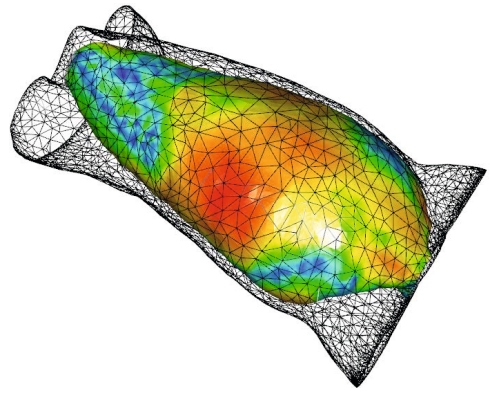

(b) Internal surface of the abdominal wall

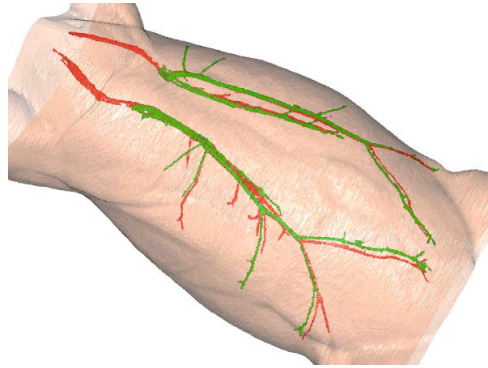

(d) Abdominal wall arteries

Fig. 3. These meshes represent the distance between our abdo-thoracic wall internal surface estimation and the ground truth using the scale from Tab. 1. On the lower right, one can see our artery position prediction (red) and the arteries segmented after pneumoperitoneum (green) with pig skin in transparency.

pneumoperitoneum. We compute the distance between each vertex of the simulated structure surface to the closest point of the ground truth point cloud. The same method is used for the internal surface of the abdominal wall, its external surface (namely the skin) and the abdominal viscera.

The average error of our simulation on the first pig is detailed in Tab 1, Regarding the second pig, we have approximately the same results for the abdominal wall 
(3.8 $\mathrm{mm} \pm 3.3 \mathrm{~mm}$ for the internal surface and $3.4 \mathrm{~mm} \pm 3.5 \mathrm{~mm}$ for the skin), but for the abdominal viscera, we obtain a worse result $(5.7 \mathrm{~mm} \pm 4.9 \mathrm{~mm})$. A lack of realism due to the gas inside viscera distorts the results. Surface error of the viscera mainly occurs close to liver since the viscera are constrained by the pelvis. Moreover, reaching a perfect accuracy does not seem crucial for trocar insertion and the knowledge of abdominal viscera surface is enough. To visually assess the error, we display in Fig. 3 the simulated surfaces with a color code described in Tab, 1 .

\subsection{Evaluation of our Artery Position Simulation}

Contrary to previous evaluations, we decide to evaluate artery simulation by computing the distance between artery bifurcations. Indeed, providing average distance between artery surfaces will not highlight translation error along the main direction of arteries. Six bifurcation points have been selected manually in simulated and ground truth arteries, and an average error of $5.1 \mathrm{~mm}$ on pig 1 and $5.6 \mathrm{~mm}$ on pig 2 have been reported.

\section{Conclusion}

In this paper, we have presented a method to predict abdominal wall and viscera positions after pneumoperitoneum using only a preoperative medical image and realistic biomechanical parameters. Three structures are generated from the preoperative CT data and are associated to different biomechanical parameters. Then, the pneumoperitoneum is simulated by applying realistic pressure on the abdominal wall (after properly taking gravity into account in our modeling approach). An evaluation has been performed using medical CT acquisitions of two pigs before and after pneumoperitoneum, allowing us to quantitatively measure the realism of our method. Results show that our method provides very realistic simulation of skin, abdominal wall and artery, with a reported accuracy below $0.5 \mathrm{~cm}$. Although the viscera simulation is less accurate (around $0.6 \mathrm{~cm}$ on average), it can still be useful to help surgical planning of laparoscopic procedures. We point out that our model does not depend on empirical values, and thus should provide reproducible accuracy and realism. In a future work, we plan to improve viscera simulation by integrating different Young's moduli to the viscera structure. We also plan to register our simulated model in the operating room using an augmented reality technique to help surgeons during trocar positioning.

\section{References}

1. Allard, J., Cotin, S., Faure, F., Bensoussan, P.J., Poyer, F., Duriez, C., Delingette, H., Grisoni, L., et al.: Sofa-an open source framework for medical simulation. Medicine Meets Virtual Reality 15, 13-18 (2007)

2. Geraci, G., Sciumè, C., Pisello, F., Li Volsi, F., Facella, T., Modica, G.: Trocarrelated abdominal wall bleeding in 200 patients after laparoscopic cholecistectomy: Personal experience. World Journal of Gastroenterology 12(44), 7165 (2006) 
3. Kitasaka, T., Mori, K., Hayashi, Y., Suenaga, Y., Hashizume, M., Toriwaki, J.-I.: Virtual Pneumoperitoneum for Generating Virtual Laparoscopic Views Based on Volumetric Deformation. In: Barillot, C., Haynor, D.R., Hellier, P. (eds.) MICCAI 2004. LNCS, vol. 3217, pp. 559-567. Springer, Heidelberg (2004)

4. Lam, A., Kaufman, Y., Khong, S.Y., Liew, A., Ford, S., Condous, G.: Dealing with complications in laparoscopy. Best Practice \& Research Clinical Obstetrics \& Gynaecology 23(5), 631-646 (2009)

5. Mori, K., Kito, M., Kitasaka, T., Misawa, K., Fujiwara, M.: Patient-specific laparoscopic surgery planning system based on virtual pneumoperitoneum technique. International Journal of Computer Assisted Radiology and Surgery 4, S140-S142 (2009)

6. Oda, M., Di Qu, J., Nimura, Y., Kitasaka, T., Misawa, K., Mori, K.: Evaluation of deformation accuracy of a virtual pneumoperitoneum method based on clinical trials for patient-specific laparoscopic surgery simulator. In: Proceedings of SPIE, vol. 8316, p. 83160G (2012)

7. Saber, A.A., Meslemani, A.M., Davis, R., Pimentel, R.: Safety zones for anterior abdominal wall entry during laparoscopy: a ct scan mapping of epigastric vessels. Annals of Surgery 239(2), 182 (2004)

8. Samur, E., Sedef, M., Basdogan, C., Avtan, L., Duzgun, O.: A robotic indenter for minimally invasive characterization of soft tissues. International Congress Series, vol. 1281, pp. 713-718. Elsevier (2005)

9. Sanchez-Margallo, F.M., Moyano-Cuevas, J.L., Latorre, R., Maestre, J., Correa, L., Pagador, J.B., Sanchez-Peralta, L.F., Sanchez-Margallo, J.A., Usan-Gargallo, J.: Anatomical changes due to pneumoperitoneum analyzed by mri: an experimental study in pigs. Surg. Radiol. Anat. 33(5), 389-396 (2011)

10. Shamiyeh, A., Wayand, W.: Laparoscopic cholecystectomy: early and late complications and their treatment. Langenbeck's Archives of Surgery 389(3), 164-171 (2004)

11. Soler, L., Marescaux, J.: Patient-specific surgical simulation. World Journal of Surgery 32(2), 208-212 (2008)

12. Song, C., Alijani, A., Frank, T., Hanna, G.B., Cuschieri, A.: Mechanical properties of the human abdominal wall measured in vivo during insufflation for laparoscopic surgery. Surgical Endoscopy 20(6), 987-990 (2006)

13. Whiteley, J.P.: The solution of inverse non-linear elasticity problems that arise when locating breast tumours. Journal of Theoretical Medicine 6(3), 143-149 (2005) 